\title{
Health-Related Quality of Life Changes in Prostate Cancer Patients after Radical Prostatectomy: A Longitudinal Cohort Study
}

\author{
Dong Wook Shin, MD, DrPH, MBA ${ }^{1,2}$ \\ Sang Hyub Lee, MD, PhD ${ }^{3}$ \\ Tae-Hwan Kim, MD, PhD ${ }^{4}$ \\ Seok Joong Yun, MD, PhD 5 \\ Jong Kil Nam, MD, $\mathrm{PhD}{ }^{6}$ \\ Seung Hyun Jeon, MD, $\mathrm{PhD}^{3}$ \\ Seung Chol Park, MD, $\mathrm{PhD}^{7}$ \\ Seung II Jung, MD, PhD ${ }^{8}$ \\ Jong-Hyock Park, MD, PhD, \\ Jinsung Park, MD, PhD'11
}

*A list author's affiliations appears at the end of the paper.
Correspondence: Jinsung Park, MD, PhD

Department of Urology, Eulji University

Hospital, Eulji University School of Medicine,

95 Dunsanseoro, Seo-gu, Daejeon 35233, Korea

Tel: 82-42-611-3533

Fax: 82-42-259-1111

E-mail: jspark.uro@gmail.com

Received April 3, 2018

Accepted July 13, 2018

Published Online July 16, 2018

*Dong Wook Shin and Sang Hyub Lee contributed equally to this work.

\begin{abstract}
Purpose
Health-related quality of life (HRQOL) information related to radical prostatectomy (RP) is valuable for prostate cancer (PC) patients needing to make treatment decisions. We aimed to investigate HRQOL change in PC patients who underwent three types of RP (open, laparoscopic, or robotic) and compared their HRQOL with that of general population.
\end{abstract}

\section{Materials and Methods}

Patients were prospectively recruited between October 2014 and December 2015. European Organization for Research and Treatment of Cancer Quality-of-Life Questionnaire (EORTC QLQ-C30) and PC-specific module (PR25) were administered before surgery (baseline) and at postoperative 3 and 12 months. At each time point, HRQOL was compared, and a difference of 10 out of 0-100 scale was considered clinically significant.

\section{Results}

Among 258 screened patients, 209 (41 open, 63 laparoscopic, and 105 robotic surgeries) were included. Compared to baseline, physical, emotional, and cognitive functioning improved at 12 months. Role functioning worsened at 3 months, but recovered to baseline at 12 months. Pain, insomnia, diarrhea, and financial difficulties also significantly improved at 12 months. Most PR25 scales excluding bowel symptoms deteriorated at 3 months. Urinary symptoms and incontinence aid recovered at 12 months, whereas sexual activity and sexual function remained poor at 12 months. Clinically meaningful differences in HRQOL were not observed according to RP modalities. Compared to the general population, physical and role functioning were significantly lower at 3 months, but recovered by 12 months. Social functioning did not recover.

\section{Conclusion}

Most HRQOL domains showed recovery within 12 months after RP, excluding sexual functioning and social functioning. Our findings may guide patients considering surgical treatment for PC.

\section{Introduction}

Prostate cancer $(\mathrm{PC})$ is a highly prevalent disease that constitutes a significant proportion of cancer death and has a profound public health impact in Western countries [1]. Similarly, increasingly westernized diet habits, increased PC screening, and a rapidly aging population have contributed

\section{Key words}

Health-related quality of life, Prostate neoplasms,

Radical prostatectomy, Social function to greater PC prevalence in Asian countries including Korea. According to 2014 nationwide cancer statistics in Korea, PC is the fifth most common cancer in men and the most common urologic tumor in Korea, with 9,785 new cases reported in 2014 [2].

The number of PC patients diagnosed in early stages has been increasing mainly due to the PC screening test, although its clinical utility remains controversial [3]. Among treatment 
modalities, surgery is still the most commonly used for clinically localized PC, and radical prostatectomy (RP) cases have significantly increased over time in Korea, from $22.4 \%$ of active treatments in 2003 to $45.4 \%$ in 2013 [4]. Meanwhile, owing to the early detection and treatment of PC, long-term survival after RP has been increasing. Therefore, healthrelated quality of life (HRQOL) has become a critical issue for PC patients undergoing RP. HRQOL information related to RP may be particularly valuable for PC patients needing to make decisions about surgery vs. active surveillance or surgery vs. radiotherapy [5]. Furthermore, the association between patient quality of life (QOL) and different surgical techniques has become another important issue. Currently, minimally invasive surgery such as robot-assisted radical prostatectomy (RARP) and laparoscopic radical prostatectomy (LRP) is widely performed, along with open retropubic radical pro-statectomy (RRP). While all surgical modalities are reported to have comparable oncologic outcomes [6], a systematic review indicated that no surgical approach was superior to the others in terms of functional outcomes [7], although several studies have reported that LRP or RARP showed better functional outcomes compared to RRP [8].

While many studies have addressed QOL after definitive therapy for localized PC $[9,10]$, no study has yet compared HRQOL based on validated QOL measurement tools among patients treated with three different surgical modalities. In this study, we aimed to investigate longitudinal changes in HRQOL of PC patients after each type of radical surgery and to compare their HRQOL with the age-matched general population.

\section{Materials and Methods}

\section{Study design and population}

This study was a multicenter, prospective longitudinal cohort study that included consecutive newly diagnosed PC patients who underwent RP (open, laparoscopic, and robotic) between October 2014 and December 2015. Patients were recruited at seven academic medical centers in Korea. Since patients' QOL after RP can be affected by the level of surgical skill, we included patients treated by surgeons who had completed an uro-oncological fellowship, had more than 8 years of uro-oncology clinical experience after their fellowship, and had a minimum of 30 cases of RP per year. All participating surgeons had performed at least 100 cases of prior RPs. Surgery method was determined after shared treatment decision making between clinician and patients. Patients who provided written informed consent were asked to fill out a self- administered questionnaire before surgery (after histologic confirmation and within 2 weeks of the date of surgery) and at 3 and 12 months postoperatively (with a window of 14 days). In the event of any difficulties understanding or completing the questions, nurse or research assistants assisted in the completion of the questionnaire.

Exclusion criteria included those patients (1) diagnosed with double primary cancer before or after PC; (2) with severe complications (grade 3 or higher according to the modified Clavien system); (3) who were undergoing adjuvant hormonal or radiation treatment; (4) who underwent salvage treatment due to biochemical recurrence; or (5) who refused to participate in the study or had communicational difficulties.

To compare the HRQOL levels of PC patients with those of general population, we used HRQOL data from the general population without a cancer history that had been collected previously $[11,12]$. The general population sample was selected from the nationwide survey, "Awareness of Quality of Cancer Treatment among the General Population in Korea," conducted between November and December in 2012. The questionnaire included the European Organization for Research and Treatment of Cancer Quality-of-Life Questionnaire (EORTC QLQ-C30), as well as the sociodemographic and medical information of the participants, making it comparable to the PC patient cohort. To ensure representativeness of the sample, a stratified probability sampling using a two-stage systematic sampling method was employed-after stratifying the population based on region, we systematically extracted samples based on the population ratio. The sampling error within a $95 \%$ confidence interval was $\pm 2.2 \%$. The participants were limited to those who were within an age range of 40-70 years (considering the purpose of the study) and those without a history of cancer.

Professionally trained interviewers visited the identified households and enrolled eligible participants. Absence of cancer history was confirmed by self-report. Among 4,851 eligible participants, a total of 2,000 individuals completed the survey questionnaire (response rate, $41.2 \%$ ). The major reasons for refusal to participation were lack of time (54\%), privacy concerns $(25 \%)$, and inconvenience (17\%). Details of the study design and procedures are described in detail elsewhere $[11,12]$.

\section{Measures of QOL}

In this outcome study, HRQOL was the primary outcome of interest. The Korean version of the EORTC QLQ-C30 $[13,14]$ and its prostate module (QLQ-PR25) $[15,16]$ were used to measure HRQOL. EORTC QLQ-C30 $[13,14]$ was designed as a multidimensional assessment of QOL using five scales of functional assessment (physical, role, emo- 
tional, cognitive, and social), three symptom scales (fatigue, nausea and vomiting, and pain), a global health status and QOL scale, and six single items (dyspnea, insomnia, appetite loss, constipation, diarrhea, and financial difficulties). Of the 30 items in QLQ-C30, 28 are scored on 4-point Likert scales, while the two items for the global QOL scale are scored on 7-point linear analog scales. The EORTC QLQ-PR25, which was designed to assess PC-specific QOL, contains 25 questions in six domains (urinary symptoms, incontinence aid, bowel symptoms, hormonal treatment-related symptoms, sexual activity, and sexual functioning) $[15,16]$. Domain scores of the QLQ-C30 and PR25 modules were calculated according to the established scoring manual provided by the EORTC QOL group as follows $[13,15]$ : the raw scores of each scales were linearly transformed to a scale of 0 to 100 , with 100 representing the worst symptom status or the best functional status, and scale scores were not computed when $>50 \%$ of the responses of the corresponding scale were missing values.

In addition, questionnaire items included sociodemo-

Table 1. Baseline characteristics of prostate cancer patients $(\mathrm{n}=209)$

\begin{tabular}{|c|c|c|c|c|c|}
\hline Variable & $\begin{array}{c}\text { Total } \\
(\mathrm{n}=209)\end{array}$ & $\begin{array}{l}\text { Open } \\
(n=41)\end{array}$ & $\begin{array}{c}\text { Laparoscopic } \\
(\mathrm{n}=63)\end{array}$ & $\begin{array}{l}\text { Robot } \\
(n=105)\end{array}$ & p-value \\
\hline Age $(\mathrm{yr})$ & $66.6 \pm 6.6$ & $67.6 \pm 6.1$ & $67.5 \pm 6.2$ & $65.7 \pm 6.9$ & 0.124 \\
\hline Height $(\mathrm{cm})$ & $166.7 \pm 5.6$ & $166.5 \pm 6.3$ & $167.0 \pm 5.5$ & $166.9 \pm 5.4$ & 0.881 \\
\hline Weight (kg) & $66.8 \pm 8.4$ & $66.9 \pm 9.1$ & $66.5 \pm 8.6$ & $67.0 \pm 8.0$ & 0.931 \\
\hline Comorbidity, any & $118 \pm 56.5$ & $26 \pm 63.4$ & $35 \pm 55.6$ & $57 \pm 54.3$ & 0.598 \\
\hline \multicolumn{6}{|l|}{ Education level } \\
\hline Less than high school & $112(53.9)$ & $27(65.9)$ & $37(58.7)$ & $48(46.2)$ & 0.065 \\
\hline High school and above & $96(46.1)$ & $14(34.1)$ & $26(41.3)$ & $56(53.8)$ & \\
\hline \multicolumn{6}{|l|}{ Marital status } \\
\hline Married & $187(89.5)$ & $39(95.1)$ & $53(84.1)$ & $95(90.5)$ & 0.181 \\
\hline Unmarried & $22(10.5)$ & $2(4.9)$ & $10(15.9)$ & $10(9.5)$ & \\
\hline \multicolumn{6}{|l|}{ Smoking } \\
\hline Current & $22(10.6)$ & $3(7.3)$ & $7(11.1)$ & $12(11.5)$ & 0.386 \\
\hline Past & $120(57.7)$ & $24(58.5)$ & $31(49.2)$ & $65(62.5)$ & \\
\hline None & $66(31.7)$ & $14(34.2)$ & $25(39.7)$ & $27(26.0)$ & \\
\hline \multicolumn{6}{|l|}{ Drinking } \\
\hline Current & $84(40.2)$ & $16(39.0)$ & $20(31.8)$ & $48(45.7)$ & 0.349 \\
\hline Past & $69(33.0)$ & $14(34.2)$ & $21(33.3)$ & $34(32.4)$ & \\
\hline None & $56(26.8)$ & $11(26.8)$ & $22(34.9)$ & $23(21.9)$ & \\
\hline \multicolumn{6}{|l|}{ Pathologic T category } \\
\hline 2 & $153(73.2)$ & $29(70.7)$ & $40(63.5)$ & $84(80.0)$ & 0.060 \\
\hline 3 & $56(26.8)$ & $12(29.3)$ & $23(36.5)$ & $21(20.0)$ & \\
\hline \multicolumn{6}{|l|}{ Pathologic $N$ category } \\
\hline 0 & $100(47.9)$ & $27(65.9)$ & $29(46.03)$ & $44(41.9)$ & 0.053 \\
\hline 1 & $3(1.4)$ & 0 & 0 & $3(2.9)$ & \\
\hline $\mathrm{x}$ & $106(50.7)$ & $14(34.1)$ & $34(53.97)$ & $58(55.2)$ & \\
\hline \multicolumn{6}{|l|}{ Pathologic Gleason score } \\
\hline 6 & 41 (19.6) & $6(14.6)$ & $15(23.8)$ & $20(19.1)$ & 0.795 \\
\hline 7 & $130(62.2)$ & $26(63.4)$ & $37(58.7)$ & $67(63.8)$ & \\
\hline$\geq 8$ & 38 (18.2) & $9(22.0)$ & $11(17.5)$ & $18(17.1)$ & \\
\hline PSA (ng/mL) & $10.4 \pm 11.9$ & $8.6 \pm 7.1$ & $10.9 \pm 9.5$ & $10.7 \pm 14.5$ & 0.578 \\
\hline \multicolumn{6}{|c|}{ Karnofsky performance status } \\
\hline 100 & $159(76.4)$ & $21(52.5)$ & $63(100)$ & 75 (71.4) & $<0.001$ \\
\hline 90 & 47 (22.6) & $17(42.5)$ & 0 & $30(28.6)$ & \\
\hline 80 & $2(1.0)$ & $2(5.0)$ & 0 & 0 & \\
\hline
\end{tabular}

Values are presented as mean \pm standard deviation or number (\%). Numbers may not sum to total number due to missing responses. p-values were calculated by chi-square test or Fisher exact test. PSA, prostate-specific antigen. 
Table 2. Health-related quality of life of prostatic cancer patients after radical prostatectomy over 1 year

\begin{tabular}{|c|c|c|c|c|c|c|}
\hline & \multirow{2}{*}{$\frac{\text { Baseline }(\mathrm{n}=209)}{\text { Mean } \pm \text { SD }}$} & \multicolumn{2}{|c|}{3 Months ( $\mathrm{n}=180)$} & \multicolumn{3}{|c|}{12 Months (n=190) } \\
\hline & & Mean \pm SD & $\begin{array}{c}\text { p-value } \\
\text { (vs. baseline) }\end{array}$ & Mean \pm SD & $\begin{array}{c}\text { p-value } \\
\text { (vs. baseline) }\end{array}$ & $\begin{array}{c}\text { p-value } \\
\text { (vs. } 3 \text { months) }\end{array}$ \\
\hline \multicolumn{7}{|l|}{ QLQ-C30 functioning scale } \\
\hline Physical function & $85.5 \pm 13.6$ & $84.7 \pm 13.5$ & 0.293 & $87.7 \pm 13.4$ & 0.039 & $<0.001$ \\
\hline Role function & $89.0 \pm 17.3$ & $85.8 \pm 20.5$ & 0.013 & $88.4 \pm 18.6$ & 0.630 & 0.052 \\
\hline Emotional function & $84.0 \pm 18.2$ & $86.5 \pm 17.5$ & 0.485 & $88.2 \pm 15.3$ & 0.002 & 0.360 \\
\hline Cognitive function & $84.9 \pm 16.4$ & $88.6 \pm 15.5$ & 0.019 & $88.4 \pm 14.8$ & 0.007 & 0.781 \\
\hline Social function & $81.7 \pm 22.1$ & $82.3 \pm 21.6$ & 0.962 & $85.0 \pm 19.0$ & 0.080 & 0.085 \\
\hline Global quality of life & $62.2 \pm 21.7$ & $62.1 \pm 18.6$ & 0.977 & $64.4 \pm 20.9$ & 0.234 & 0.041 \\
\hline \multicolumn{7}{|l|}{ QLQ-C30 symptom scale } \\
\hline Fatigue & $20.6 \pm 17.9$ & $23.1 \pm 17.9$ & 0.103 & $20.3 \pm 17.9$ & 0.968 & 0.154 \\
\hline Nausea and vomiting & $4.9 \pm 11.5$ & $4.2 \pm 10.1$ & 0.629 & $4.1 \pm 11.6$ & 0.749 & 0.927 \\
\hline Pain & $14.0 \pm 20.5$ & $11.8 \pm 14.6$ & 0.459 & $8.8 \pm 15.9$ & 0.001 & 0.003 \\
\hline Dyspnea & $11.2 \pm 18.9$ & $9.3 \pm 17.6$ & 0.500 & $10.7 \pm 19.8$ & 0.632 & 0.773 \\
\hline Insomnia & $18.2 \pm 24.9$ & $18.9 \pm 26.6$ & 0.578 & $14.4 \pm 23.1$ & 0.025 & 0.053 \\
\hline Appetite loss & $11.3 \pm 18.9$ & $14.6 \pm 22.3$ & 0.041 & $8.8 \pm 16.6$ & 0.085 & $<0.001$ \\
\hline Constipation & $14.4 \pm 22.1$ & $12.2 \pm 19.6$ & 0.512 & $13.4 \pm 22.8$ & 0.742 & 0.781 \\
\hline Diarrhea & $10.5 \pm 17.8$ & $5.6 \pm 15.7$ & 0.002 & $6.1 \pm 15.7$ & 0.007 & 0.527 \\
\hline Financial difficulties & $21.7 \pm 26.7$ & $17.7 \pm 24.1$ & 0.095 & $14.6 \pm 21.1$ & 0.002 & 0.290 \\
\hline \multicolumn{7}{|l|}{ QLQ-PR25 scale } \\
\hline Urinary symptom & $22.2 \pm 16.8$ & $29.2 \pm 16.7$ & $<0.001$ & $22.0 \pm 16.8$ & 0.896 & $<0.001$ \\
\hline Incontinence aid (conditional) & $12.0 \pm 22.3$ & $28.9 \pm 27.2$ & $<0.001$ & $17.9 \pm 30.7$ & 1.000 & 0.005 \\
\hline Bowel symptoms & $7.7 \pm 10.7$ & $6.9 \pm 10.4$ & 0.877 & $5.3 \pm 9.3$ & 0.007 & 0.006 \\
\hline Hormonal treatment-related symptom & $9.2 \pm 10.2$ & $12.0 \pm 8.9$ & $<0.001$ & $11.3 \pm 9.4$ & 0.010 & 0.132 \\
\hline Sexual activity & $32.6 \pm 27.7$ & $17.7 \pm 25.8$ & $<0.001$ & $27.0 \pm 27.1$ & 0.035 & $<0.001$ \\
\hline Sexual function (conditional) & $63.1 \pm 23.8$ & $45.0 \pm 26.4$ & $<0.001$ & $44.0 \pm 23.7$ & $<0.001$ & 0.328 \\
\hline
\end{tabular}

SD, standard deviation; QLQ, Quality-of-Life Questionnaire.

graphic information such as marital status, education level, and smoking and drinking status. Clinical data including the type of surgery, tumor stage, Gleason score, Karnofsky performance status (KPS), and initial prostate-specific antigen (PSA) value were obtained from hospital medical records.

\section{Statistical analysis}

Descriptive statistics were used for the baseline demographic and clinical characteristics. Mean (standard deviation) of each scale of the EOTRC QLQ-C30 and the PR25 module at each time point was calculated.

For the comparisons of each domain of HRQOL by time point and operation type, we used a generalized estimating equation (GEE) to perform the analyses of repeated measures data. For the comparison of HRQOL by time point for the entire patient population, the models were adjusted for baseline sociodemographic characteristics, clinical characteristics, and types of surgery as outlined above, and comparisons were made between 3 months vs. baseline, 12 months vs. baseline, and 12 months vs. 3 months, respectively, while case wise deletion was used for any missing data. Comparisons of the changes in HRQOL according to the type of surgery were also performed using the same GEE model for both the postoperative 3-month and 12-month time points. Interactions between operation type and time point were generally not significant, and were excluded from the final models.

Function in 5 scales of the EORTC QLQ-C30 and overall QOL were compared between the PC patients and the general population using a subset of the matched population. For matching, we used 1:1 propensity score matching, in order to reduce bias in the statistical analysis of observational data. Considering the age distribution of the general population dataset, we excluded those who were aged under 40 or over 71 from each dataset. Then, propensity scores were estimated using logistic regression based on baseline age, marital status, education level, and smoking and drinking status and matched with a Mahalanobis algorithm with a caliper of 0.01 [17]. Paired $t$ test was used to compare the 

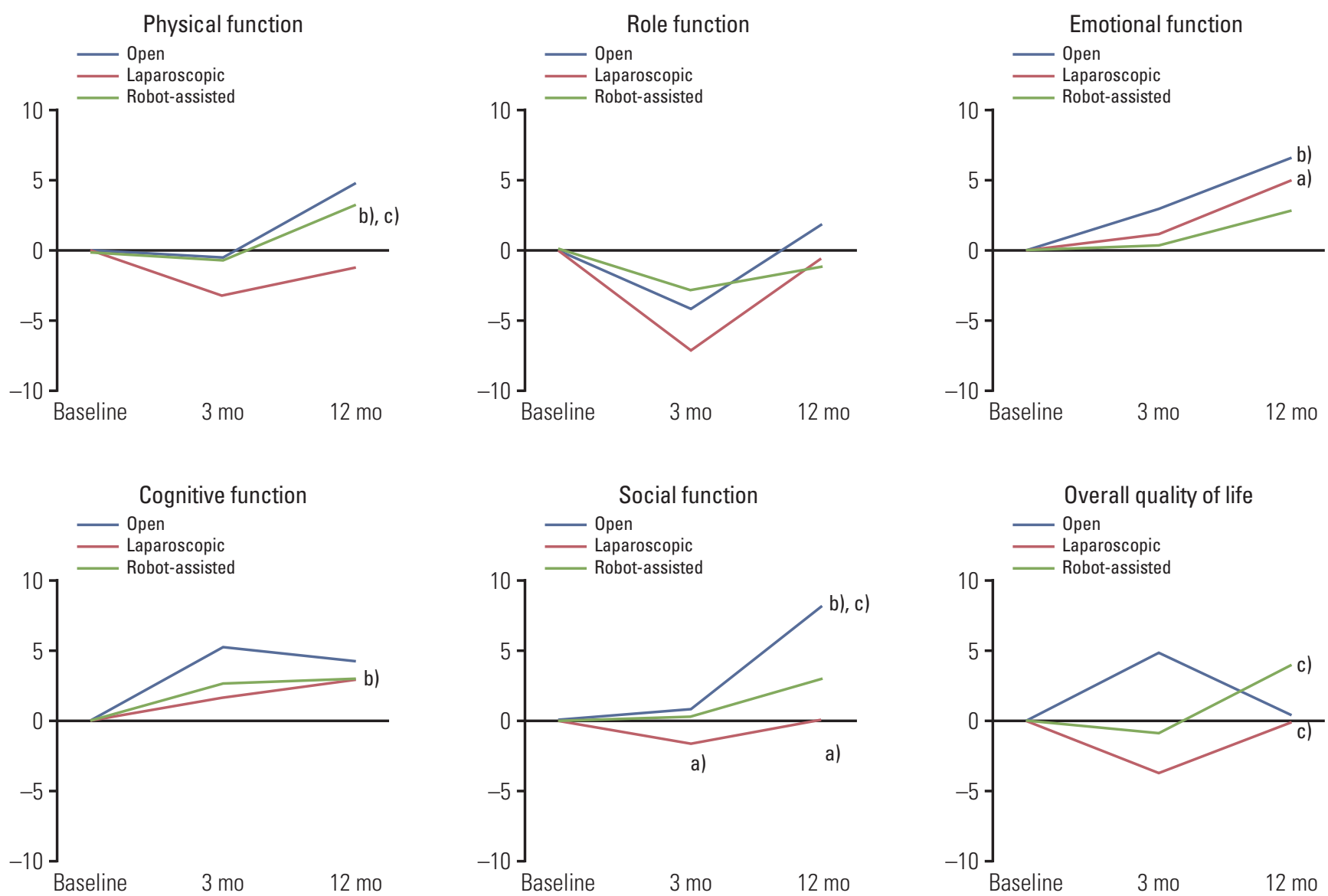

Fig. 1. Changes in the QLQ-C30 functioning scales according to the type of radical prostatectomy (RP). ${ }^{a}$ Significant in comparison between laparoscopic or robotic RP and open RP at the same time point (either postoperative 3 or 12 months), bignificant in comparison with baseline status within each group, ${ }^{c}$ Significant in comparison with status at postoperative 3 months within each group.

functional scales of the HRQOL between the PC patients and the general population for each time point.

As this was an observational study, sample size was not formally determined, and it was based on enrollment volume of participating centers. All analyses were conducted using Stata ver. 14.0 (StataCorp, College Station, TX) with $p<0.05$ considered statistically significant. A clinically meaningful difference was defined as 10 points or more on the 0-100 scale of the EORTC QLQ scores, as previously suggested [18].

\section{Ethical statement}

The Institutional Review Board of the National Cancer Center of Korea approved this general population survey (IRB No. NCCNCS-12-635) and the study protocol was approved by the Institutional Review Board of the Eulji University Hospital (No. 2014-08-012).

All participants were fully informed as to the purpose of the study and provided written consent.

\section{Results}

\section{Subjects and recruitment results}

During the study period, a total of 258 patients were recruited. After excluding 13 patients who did not meet the inclusion criteria and 36 patients with various exclusion criteria, 209 patients constituted the PC population. The study flow is shown in $\mathrm{S} 1$ Fig. All patients finished the baseline survey, and $180(86.1 \%)$ and $190(90.9 \%)$ patients completed the questionnaires at postoperative 3 and 12 months, respectively. 

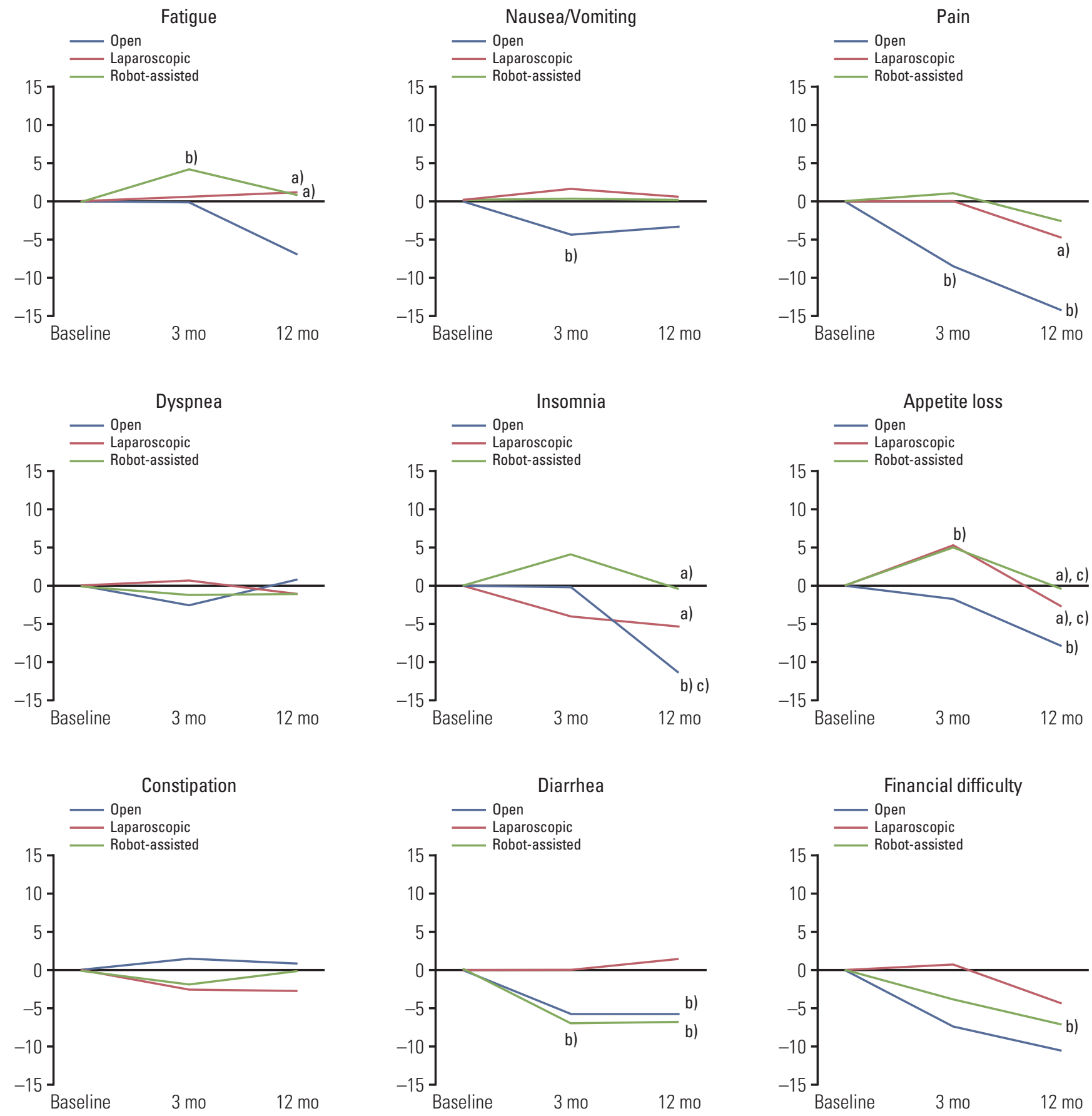

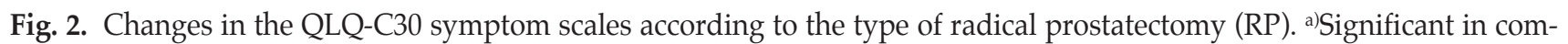
parison between laparoscopic or robotic RP and open RP at the same time point (either postoperative 3 or 12 months), b)Significant in comparison with baseline status within each group, ') Significant in comparison with status at postoperative 3 months within each group. 

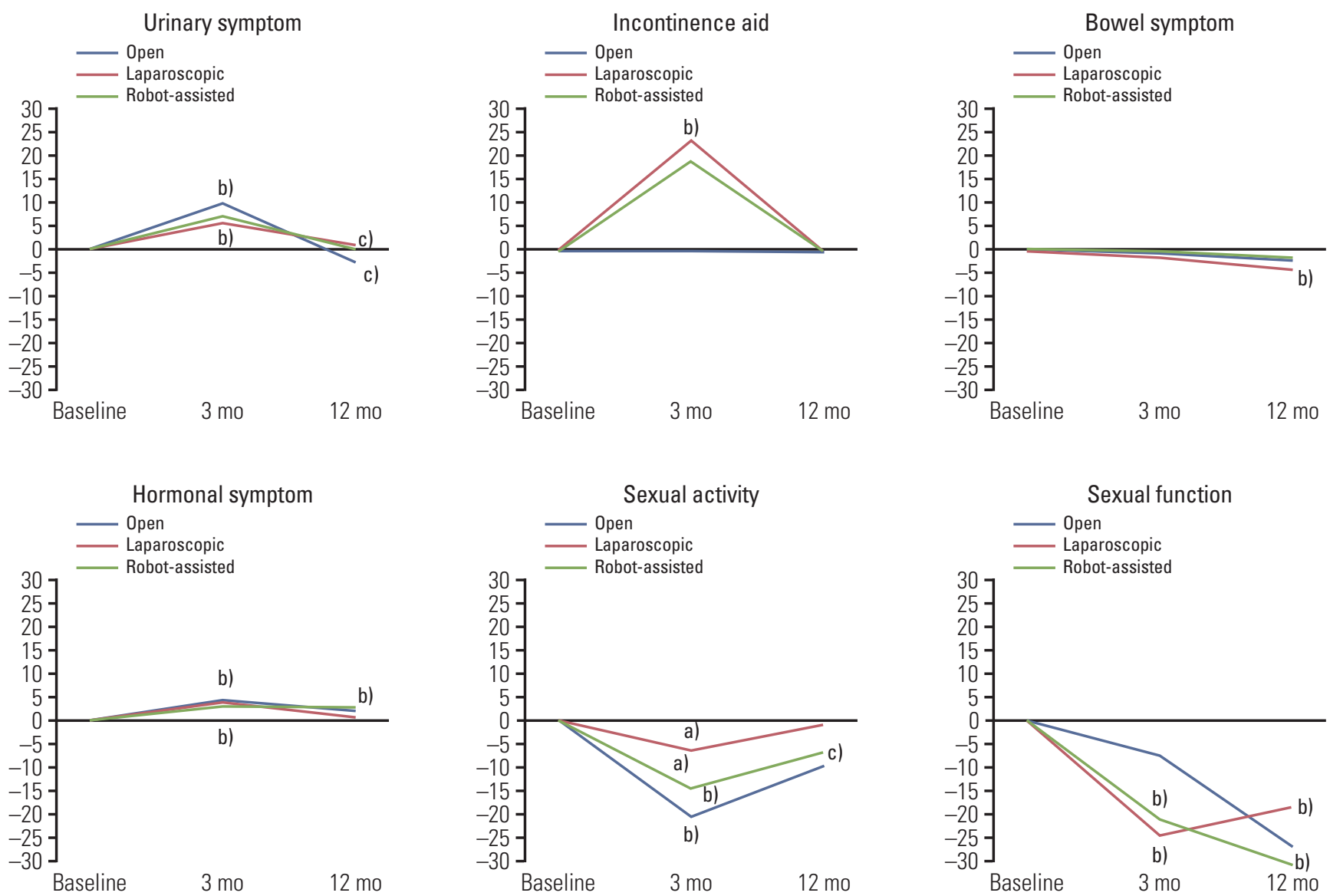

Fig. 3. Changes in the QLQ-PR25 module according to the type of radical prostatectomy (RP). a)Significant in comparison between laparoscopic or robotic RP and open RP at the same time point (either postoperative 3 or 12 months), b)Significant in comparison with baseline status within each group, ') Significant in comparison with status at postoperative 3 months within each group.

\section{Patient characteristics}

The number of patients who underwent RRP, LRP, and RARP was $41(19.6 \%), 63(30.1 \%)$, and $105(50.2 \%)$, respectively. Table 1 summarizes the baseline characteristics of the PC patients. Mean age of patients was 66.6 years (standard deviation [SD], 6.6). Mean PSA level was $10.4 \mathrm{ng} / \mathrm{mL}$ (SD, 11.9; range, 1.2 to 118). According to the pathologic stage, 153 $(73.2 \%)$ and $56(26.8 \%)$ patients had pathological T2 and T3 disease, respectively. The number of patients with pathological Gleason scores of 6, 7, and 8 or higher was $41(19.6 \%)$, $130(62.2 \%)$, and $38(18.2 \%)$, respectively. The majority of patients $(76.4 \%)$ had a normal performance status. No significant differences were observed in baseline characteristics among the three RP groups, except performance status (Table 1).

\section{Overall trends in QOL of PC patients}

QOL changes in the PC patients after RP are shown in Table 2. Most of the HRQOL categories assessed by the EORTC QLQ-C30 showed improvement over time. While role functioning worsened at postoperative 3 months, it recovered over time to nearly baseline status at 12 months. On the other hand, cognitive functioning significantly improved at 3 months compared to baseline, and the improvement was maintained at 12 months. At 12 months, physical, emotional, and cognitive functioning showed significant improvement compared to baseline. While global QOL at 3 months was similar compared to baseline, it significantly improved at 12 months. Similarly, some of the QLQ-C30 symptom scores improved over time. Pain, insomnia, diarrhea, and financial difficulties showed significant improvement at 12 months compared to baseline. Meanwhile, 
Table 3. Baseline characteristics of prostate cancer patients and general population control: Before and After Propensity Score matching

\begin{tabular}{|c|c|c|c|c|c|c|}
\hline & \multicolumn{3}{|c|}{ Unmatched sample } & \multicolumn{3}{|c|}{ Matched sample } \\
\hline & PC patients & Control & p-value & PC patients & Control & p-value \\
\hline Total & $156(100)$ & $623(100)$ & & $114(100)$ & $114(100)$ & \\
\hline Age (yr) & $63.9 \pm 5.4$ & $57.4 \pm 5.7$ & $<0.001$ & $62.7 \pm 5.5$ & $62.1 \pm 5.2$ & 0.359 \\
\hline Comorbidity, any & $90(57.7)$ & $221(35.5)$ & $<0.001$ & $60(52.6)$ & $62(54.4)$ & 0.791 \\
\hline Education level, high school and above & $79(60.0)$ & $496(79.6)$ & $<0.001$ & $58(50.9)$ & $60(52.6)$ & 0.791 \\
\hline Marital status, married & $141(90.4)$ & $600(96.3)$ & $<0.001$ & $106(93)$ & $108(94.7)$ & 0.581 \\
\hline Smoking, current & $18(11.6)$ & $328(52.7)$ & $<0.001$ & $18(15.8)$ & $18(15.8)$ & 1.000 \\
\hline Drinking, current & $70(44.9)$ & $508(81.5)$ & $<0.001$ & $64(56.1)$ & $59(51.8)$ & 0.506 \\
\hline \multicolumn{7}{|l|}{ Pathologic $\mathrm{T}$ category } \\
\hline 2 & $117(75.0)$ & - & - & $84(73.7)$ & - & - \\
\hline 3 & $39(25.0)$ & - & - & $30(26.3)$ & - & - \\
\hline \multicolumn{7}{|l|}{ Pathologic $\mathbf{N}$ category } \\
\hline 0 & $70(44.9)$ & - & - & $57(50.0)$ & - & - \\
\hline 1 & $3(1.9)$ & - & - & $2(1.8)$ & - & - \\
\hline $\mathrm{x}$ & $83(53.2)$ & - & - & $55(48.2)$ & - & - \\
\hline \multicolumn{7}{|l|}{ Pathologic Gleason score } \\
\hline 6 & $31(19.9)$ & - & - & $24(21.1)$ & - & - \\
\hline 7 & $98(62.8)$ & - & - & $71(62.3)$ & - & - \\
\hline$\geq 8$ & $27(17.3)$ & - & - & 19 (16.6) & - & - \\
\hline Prostate-specific antigen $(\mathrm{ng} / \mathrm{mL})$ & $9.98 \pm 9.77$ & - & - & $9.76 \pm 9.16$ & - & - \\
\hline \multicolumn{7}{|l|}{ Karnofsky performance status } \\
\hline 100 & $119(76.8)$ & - & - & $83(73.5)$ & - & - \\
\hline 90 & $34(21.9)$ & - & - & $28(24.8)$ & - & - \\
\hline 80 & $2(1.3)$ & - & - & $2(1.8)$ & - & - \\
\hline \multicolumn{7}{|l|}{ Type of surgery } \\
\hline Open & $28(18.0)$ & - & - & $22(19.3)$ & - & - \\
\hline Laparoscopic & $46(29.5)$ & - & - & $27(23.7)$ & - & - \\
\hline Robot & $82(52.6)$ & - & - & $65(57.0)$ & - & - \\
\hline
\end{tabular}

Values are presented as number (\%) or mean \pm standard deviation.

fatigue, nausea and vomiting, dyspnea, and constipation showed no significant change before and after RP.

PC-specific HRQOL, as assessed by EORTC QLQ-PR25, showed significant deterioration with the exception of bowel symptoms at postoperative 3 months compared to baseline (Table 2). Urinary symptoms and incontinence aid significantly worsened at 3 months, though they recovered at 12 months to nearly baseline status. Bowel symptoms significantly improved at 12 months compared to baseline. However, hormonal treatment-related symptoms, sexual activity, and sexual functioning were significantly worse at 3 months and remained poorer than baseline at 12 months, although they showed a tendency toward improvement after 3 months.

\section{Changes in QOL by type of RP}

While the QLQ-C30 functional scales after each type of RP (Fig. 1) generally improved over time, the recovery pattern was not significantly different according to the surgery type except in the case of emotional and social functioning. Emotional functioning at 12 months and social functioning at 3 and 12 months were significantly worse in the LRP group than in the RRP group. However, the difference was not clinically significant (score difference $<10$ ) (Fig. 1).

Fig. 2 shows the changes in the QLQ-C30 symptom scales after each type of RP. While most symptom scales were similar at each time point among the groups, the RRP group showed greater improvement compared to the RARP or LRP groups in terms of fatigue, pain, insomnia, and appetite loss at 12 months, and the score differences for pain and insomnia 

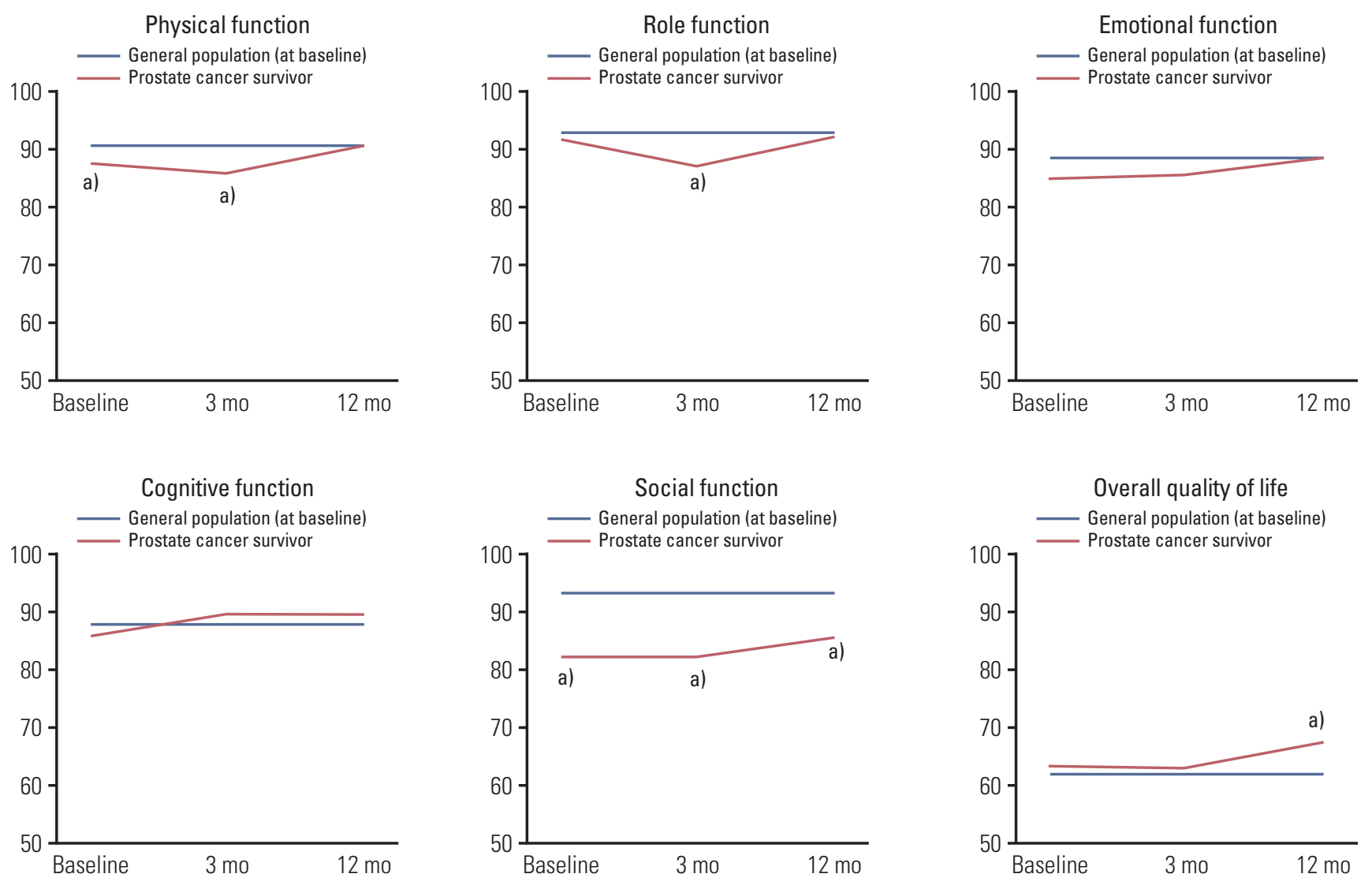

Fig. 4. Comparison of the QLQ-C30 functioning scales and overall quality of life between matched prostate cancer patients and general population control. a)Significant in comparison with the baseline values of the general population control.

were clinically significant (> 10 point).

Comparison of the QLQ-PR25 among the RP groups (Fig. 3) showed that while 3 of the RP modalities showed a similar recovery pattern, sexual activity at 3 months was significantly better in the LRP and RARP groups than in the RRP group.

\section{Comparison of QOL with matched general population}

After propensity-score matching, 114 patients were selected and compared with the same number of controls from the general population. Table 3 shows the baseline characteristics of PC patients and matched general population. There were no statistical differences between the two groups regarding age, comorbidity, education level, marital status, smoking, and drinking.

Comparisons of the HRQOL changes over time between PC patients after RP and the matched population are shown in Fig. 4. At baseline, the physical and social functioning of PC patients was significantly lower than that of the general population, with no improvement at 3 months after surgery. While the physical functioning of the PC patients became similar to that of the general population at postoperative 12 months, the social functioning of the PC patients continued to be significantly lower than that of the general population at 12 months. Role, emotional, and cognitive functioning, as well as the overall QOL of the PC patients were similar to those of the general population at baseline. While the role functioning of the PC patients significantly decreased at postoperative 3 months, it recovered and became similar to that of the general population at 12 months. The emotional and cognitive functioning of the PC patients showed no change over 12 months and remained comparable to that of the general population. On the other hand, the overall QOL of the PC patients at postoperative 12 months was significantly better than that of the general population. 


\section{Discussion}

To our knowledge, this is the first prospective and longitudinal cohort study to examine the changes in HRQOL after three types of RP and to compare the HRQOL of disease-free PC survivors with that of the general population. More specifically, our study is the first to compare HRQOL after RRP, LRP, and RARP using validated questionnaires.

Overall, RP did not have a negative impact on QLQ-C30 functioning scales over 1 year, except for a transient decrease in role functioning at 3 months that recovered to baseline status at 12 months. In addition, physical, emotional, and cognitive functioning and global QOL showed significant improvement over 1 year without deterioration at 3 months. Our results, showing no negative impact on the functional scales after RP, are consistent with those of a prior German study using EORTC QLQ-C30 and QLQ-PR25 [19] and a U.S. study using SF-36 and UCLA questionnaire [20]. For example, a longitudinal study by Litwin et al. [20] reported that over $90 \%$ of RP patients reached baseline HRQOL status at postoperative 1 year. Our results also show that several symptom scales, specifically pain, insomnia, diarrhea, and financial difficulties, significantly improved over time after $R P$. The finding that many domains of HRQOL at postoperative 1 year were significantly higher than those at baseline status may reflect that the baseline QOL of patients with untreated PC was compromised and subsequently improved over time after surgery, which is consistent with a prior study [20].

In contrast, $\mathrm{RP}$ was found to have a negative impact on PCspecific HRQOL. At postoperative 3 months, 5 domains of the QLQ-PR25, excluding bowel symptoms, showed significant deterioration. Urinary symptoms and incontinence aid recovered at 12 months, whereas hormonal symptoms, sexual activity, and sexual functioning did not recover to baseline status and remained poorer at 12 months. Our results are in accordance with previous studies showing that patients who underwent RP experienced lower urinary tract symptoms and sexual dysfunction, with the former recovering gradually and the latter not improving $[9,10,21]$. Indeed, a U.S. study reported that urinary function recovered at postoperative 12 months following RP, whereas sexual function did not improve [9]. Thus, group education [22] and erectile rehabilitation therapy (i.e., pills, injection, vacuum device) $[23,24]$ may be considered after surgery in order to minimize postoperative sexual dysfunction in RP patients.

We found that most functioning and symptom scales were not significantly different among the RP groups with a few exceptions. For example, emotional and social functioning was lower in patients who underwent LRP than in those who underwent RRP (not clinically significant, i.e., score differ- ence $<10$ ), while the improvements in pain and insomnia were greater (clinically significant) in the RRP group than in the LRP and RARP groups. Meanwhile, most PC-specific HRQOL categories were similar between the RP groups; however, sexual activity at 3 months (though not at 12 months) was significantly better (also clinically significant) in the LRP and RARP groups than in the RRP group. Consistent with this finding, several recent studies have reported that LRP and RARP are superior to traditional RRP in terms of urinary incontinence and sexual dysfunction complications $[7,8]$, although those studies have limitations in that the investigators interviewed patients [7] or used a non-validated questionnaire [8]. Since interview-assessed functional outcomes after surgery show a limited association with questionnairebased evaluations and may overestimate functional recovery [25], our results based on self-administered validated questionnaires that indicate comparable HRQOL outcomes among RP types may more accurately reflect QOL data in terms of patient perception.

Given that the baseline HRQOL of PC patients may be compromised due to untreated cancer and thus may differ from that of the general population, we also longitudinally compared the HRQOL of PC patients with that of the general population. We found that PC patients seem to experience a slight deterioration in physical, role, and social functioning from the time of surgery onward for several months. While physical and role functioning appeared to recover up to 12 months postoperatively, the social functioning of PC patients remained lower than that of the general population at 1 year, which merits clinical attention and intervention. Group education [22] and psychosocial intervention [26] may be helpful to improve the social functioning of PC survivors and have been found to increase the likelihood of remaining employed.

Interestingly, the overall QOL score of the PC patients was similar to that of the general population at baseline and postoperative 3 months, and became even slightly higher at postoperative 12 months, although this increase was not clinically significant. This finding was consistent with cross-sectional studies from Germany $[27,28]$ in which the global QOL of PC patients was found to be comparable to that of the general population. As in previous studies [29,30], our results indicate that PC survivors have the capacity to positively adapt to the challenges of cancer diagnosis and treatment and that their experience of cancer survival may contribute to a more positive perception of QOL.

Our study has certain limitations. We acknowledge possibility of selection bias. Though we aimed to enroll all consecutive RP patients during the study period, 18.9\% (49 out of 289 screened patients) were excluded due to various reasons (shown in the S1 Fig.), which might affect HRQOL. Though exclusion of some patients were to minimize impact of pos- 
sible confounding factors on primary outcome (HRQOL), it is possible that patients who participated in our study are generally in better physical condition and therefore have better HRQOL than those who did not participate. In addition, our results were based on a relatively short follow-up period (1 year), with a lack of randomization to treatment. Relatively small sample size, specifically in open RP group, is another limitation. Because entire patient cohort was not large enough $(n=209)$ and matched cohort with general population was rather small $(\mathrm{n}=114)$, we could show QOL comparison results between matched PC patients and general population (Fig. 4) without separate comparison between each type of RP and general population. Nevertheless, the strengths of our study include its prospective design, inclusion of a longitudinal cohort, and usage of a validated and standardized questionnaire during follow-up. Despite non-randomization of surgical methods, no significant differences in baseline characteristics among the three RP groups, except performance status (probably due to very small number of patients with Karnofsky score 80), indicate that selection bias depending on the surgical methods may be minimal. Although our patients were recruited from multiple institutions and treated by multiple surgeons, we recruited patients who were treated by experienced uro-oncologic surgeons in order to minimize the factor of surgical expertise. Finally, the comparison of the HRQOL of PC patients with that of agematched general populations is another strength.

In the present longitudinal cohort study that included both a comparison of HRQOL among the three current RP surgical modalities and a comparison of HRQOL between RP patients and the general population, the majority of the functional and symptom scales significantly improved over time after RP. In contrast, RP patients showed a transient deterioration of PC-specific HRQOL, and those with compromised PC-specific QOL experienced recovery over the course of 1 year in all scales except sexual functioning. The various surgical modalities all showed a similar recovery pattern of HRQOL. The compromised social functioning of RP patients at postoperative 1 year warrants clinical consideration and further supportive interventions. Our findings have the potential to assist patients to make truly informed decisions around surgical treatment for PC.

\section{Electronic Supplementary Material}

Supplementary materials are available at Cancer Research and Treatment website (https:// www.e-crt.org).

\section{Conflicts of Interest}

Conflict of interest relevant to this article was not reported.

\section{Acknowledgments}

This study was supported by a research fund from the Hannam Urological Association, 2015.

\section{Author Details}

${ }^{1}$ Department of Family Medicine, Samsung Medical Center, Seoul, ${ }^{2}$ Department of Digital Health, SAIHST, Sungkyunkwan University, Seoul, ${ }^{3}$ Department of Urology, Kyung Hee University School of Medicine, Seoul, ${ }^{4}$ Department of Urology, School of Medicine, Kyungpook National University, Daegu, ${ }^{5}$ Department of Urology, Chungbuk National University College of Medicine, Cheongju, ${ }^{6}$ Department of Urology, Pusan National University Yangsan Hospital, Yangsan, ${ }^{7}$ Department of Urology, Wonkwang University School of Medicine, Iksan, ${ }^{8}$ Department of Urology, Chonnam National University Medical School, Gwangju, ${ }^{9}$ College of Medicine/Graduate School of Health Science Business Convergence, Chungbuk National University, Cheongju, ${ }^{10}$ Division of Cancer Policy and Management, National Cancer Control Research Institute, National Cancer Center, Goyang, ${ }^{11}$ Department of Urology, Eulji University Hospital, Eulji University School of Medicine, Daejeon, Korea

\section{References}

1. Siegel R, Ma J, Zou Z, Jemal A. Cancer statistics, 2014. CA Cancer J Clin. 2014;64:9-29.

2. Jung KW, Won YJ, Oh CM, Kong HJ, Lee DH, Lee KH, et al. Cancer statistics in Korea: incidence, mortality, survival, and prevalence in 2014. Cancer Res Treat. 2017;49:292-305.

3. Moore AL, Dimitropoulou P, Lane A, Powell PH, Greenberg DC, Brown $\mathrm{CH}$, et al. Population-based prostate-specific antigen testing in the UK leads to a stage migration of prostate cancer. BJU Int. 2009;104:1592-8.

4. Park J, Suh B, Shin DW, Hong JH, Ahn H. Changing patterns of primary treatment in Korean men with prostate cancer over 10 years: a nationwide population based study. Cancer Res Treat. 2016;48:899-906.

5. Bergius S, Torvinen S, Muhonen T, Roine RP, Sintonen H, Taari K. Health-related quality of life among prostate cancer patients: real-life situation at the beginning of treatment. Scand 
J Urol. 2017;51:13-9.

6. Tewari A, Sooriakumaran P, Bloch DA, Seshadri-Kreaden U, Hebert AE, Wiklund P. Positive surgical margin and perioperative complication rates of primary surgical treatments for prostate cancer: a systematic review and meta-analysis comparing retropubic, laparoscopic, and robotic prostatectomy. Eur Urol. 2012;62:1-15.

7. Tewari A, Srivasatava A, Menon M; Members of the VIP Team. A prospective comparison of radical retropubic and robotassisted prostatectomy: experience in one institution. BJU Int. 2003;92:205-10.

8. Anastasiadis AG, Salomon L, Katz R, Hoznek A, Chopin D, Abbou CC. Radical retropubic versus laparoscopic prostatectomy: a prospective comparison of functional outcome. Urology. 2003;62:292-7.

9. Frank SJ, Pisters LL, Davis J, Lee AK, Bassett R, Kuban DA. An assessment of quality of life following radical prostatectomy, high dose external beam radiation therapy and brachytherapy iodine implantation as monotherapies for localized prostate cancer. J Urol. 2007;177:2151-6.

10. Sanda MG, Dunn RL, Michalski J, Sandler HM, Northouse L, Hembroff L, et al. Quality of life and satisfaction with outcome among prostate-cancer survivors. N Engl J Med. 2008;358: 1250-61.

11. Kim S, Shin DW, Yang HK, Kim SY, Ko YJ, Cho B, et al. Public perceptions on cancer incidence and survival: a nation-wide survey in Korea. Cancer Res Treat. 2016;48:775-88.

12. Yoo H, Shin DW, Jeong A, Kim SY, Yang HK, Kim JS, et al. Perceived social support and its impact on depression and health-related quality of life: a comparison between cancer patients and general population. Jpn J Clin Oncol. 2017;47:72834.

13. Aaronson NK, Ahmedzai S, Bergman B, Bullinger M, Cull A, Duez NJ, et al. The European Organization for Research and Treatment of Cancer QLQ-C30: a quality-of-life instrument for use in international clinical trials in oncology. J Natl Cancer Inst. 1993;85:365-76.

14. Yun YH, Park YS, Lee ES, Bang SM, Heo DS, Park SY, et al. Validation of the Korean version of the EORTC QLQ-C30. Qual Life Res. 2004;13:863-8.

15. van Andel G, Bottomley A, Fossa SD, Efficace F, Coens C, Guerif S, et al. An international field study of the EORTC QLQ-PR25: a questionnaire for assessing the health-related quality of life of patients with prostate cancer. Eur J Cancer. 2008;44:2418-24.

16. Park J, Shin DW, Yun SJ, Park SW, Jeon SS, Kwak C, et al. Cross-cultural application of the Korean version of the European Organization for Research and Treatment of Cancer Quality of Life Questionnaire for patients with prostate cancer: EORTC QLQ-PR25. Oncology. 2013;85:299-305.

17. Rubin DB. On principles for modeling propensity scores in medical research. Pharmacoepidemiol Drug Saf. 2004;13: 855-7.

18. Maringwa J, Quinten C, King M, Ringash J, Osoba D, Coens $C$, et al. Minimal clinically meaningful differences for the EORTC QLQ-C30 and EORTC QLQ-BN20 scales in brain cancer patients. Ann Oncol. 2011;22:2107-12.

19. Bach P, Doring T, Gesenberg A, Mohring C, Goepel M. Quality of life of patients after retropubic prostatectomy: pre- and postoperative scores of the EORTC QLQ-C30 and QLQ-PR25. Health Qual Life Outcomes. 2011;9:93.

20. Litwin MS, Melmed GY, Nakazon T. Life after radical prostatectomy: a longitudinal study. J Urol. 2001;166:587-92.

21. Resnick MJ, Koyama T, Fan KH, Albertsen PC, Goodman M, Hamilton AS, et al. Long-term functional outcomes after treatment for localized prostate cancer. N Engl J Med. 2013;368:43645.

22. Lepore SJ, Helgeson VS, Eton DT, Schulz R. Improving quality of life in men with prostate cancer: a randomized controlled trial of group education interventions. Health Psychol. 2003; 22:443-52.

23. Montorsi F, Brock G, Stolzenburg JU, Mulhall J, Moncada I, Patel HR, et al. Effects of tadalafil treatment on erectile function recovery following bilateral nerve-sparing radical prostatectomy: a randomised placebo-controlled study (REACTT). Eur Urol. 2014;65:587-96.

24. Roth AJ, Weinberger MI, Nelson CJ. Prostate cancer: psychosocial implications and management. Future Oncol. 2008;4: 561-8.

25. van der Poel HG, Tillier C, de Blok WM, Acar C, van Muilekom EH, van den Bergh RC. Interview-based versus questionnaire-based quality of life outcomes before and after prostatectomy. J Endourol. 2013;27:1411-6.

26. Parahoo K, McDonough S, McCaughan E, Noyes J, Semple C, Halstead EJ, et al. Psychosocial interventions for men with prostate cancer: a Cochrane systematic review. BJU Int. 2015; 116:174-83.

27. Zenger M, Hinz A, Stolzenburg JU, Rabenalt R, Schwalenberg T, Schwarz R. Health-related quality of life of prostate cancer patients compared to the general German population: age-specific results. Urol Int. 2009;83:166-70.

28. Bestmann B, Loetters C, Diemer T, Weidner W, Kuchler T, Rohde V. Prostate-specific symptoms of prostate cancer in a German general population. Prostate Cancer Prostatic Dis. 2007;10:52-9.

29. Peuckmann V, Ekholm O, Rasmussen NK, Moller S, Groenvold $\mathrm{M}$, Christiansen $\mathrm{P}$, et al. Health-related quality of life in long-term breast cancer survivors: nationwide survey in Denmark. Breast Cancer Res Treat. 2007;104:39-46.

30. Gotay CC, Muraoka MY. Quality of life in long-term survivors of adult-onset cancers. J Natl Cancer Inst. 1998;90:656-67. 